\title{
LA ACNDEP Y SU PAPEL POLÍTICO EN EL PRIMER FRANQUISMO
}

\author{
POR \\ CRISTINA BARREIRO ${ }^{1}$ \\ Universidad CEU-San Pablo
}

\begin{abstract}
RESUMEN
Análisis de las posiciones adoptadas por la Asociación Católica Nacional de Propagandistas (ACNdeP) y sus miembros, desde el final de la Guerra Civil hasta 1945, momento en el que concluye el conflicto mundial y Franco forma un Gobierno con destacada participación de los católicos. Se trata de comprobar cómo pese a la elevada influencia de las personalidades vinculadas a Falange en la nueva estructura del Estado, los propagandistas van cimentando posiciones en los diferentes organismos oficiales lo que les permitirá, una vez terminado el conflicto europeo, consolidarse como una de las familias políticas más relevantes del franquismo. Durante este tiempo en el que la ACNdeP estuvo presidida por Fernando Martín-Sánchez, los propagandistas resultan especialmente relevantes en los Ministerios de Justicia y Educación y se convierten en actores protagonistas de cara a la elaboración de la legislación del Estado y muy especialmente, como artífices del nuevo entramado jurídico destinado a regular las relaciones con la Iglesia. En este sentido, la labor de José Ibáñez Martín, Luis Ortíz Muñoz, Juan de Contreras, José Pemartín, Máximo Cuervo o Joaquín Ruiz-Giménez, resulta especialmente significativa.
\end{abstract}

PALABRAS CLAVE: Estado confesional; franquismo; catolicismo; influencia institucional; desarrollo político.

\section{THE ACNDEP AND HIS POLITICAL PAPER IN THE FIRST FRANCO'S REGIME}

\begin{abstract}
Analysis of the positions adopted as the ACNdeP and his members, from the end of the Civil war until 1945, moment in the one that concludes the World war and Franc forms a Government with out-standing participation of the catholics. It is a question of verifying how in spite of the high influence of the personalities linked to phalanx in the new structure of the State, the ACNdeP are establishing positions in the different official organisms what will allow them, finished the European conflict, to be consolidated once as one of the most relevant political families of the Franco's regime. During this time in which the ACNdeP was presided by Fernando Martin-Sánchez, the menmers turn out to be specially relevant in the departments of Justice and Education, beside protagonists turn into actors with a view to the production of the legislation of the State and very specially, as makers of the new juridical studding destined to regulate the relations with the Church. In this respect, the labor of Jose Ibáñez Martin, Luis Ortíz Muñoz, Juan de Contreras, Jose Pemartín, Máximo Cuervo or Joaquín Ruiz-Giménez, it turns out to be specially significant.
\end{abstract}

KEY WORDS: Confessional State; Francoism; Catholicism; Institutional influence; Political development.

CÓMO CITAR ESTE ARTíCULO / CITATION: Barreiro, C. 2018. "La ACNdeP y su papel político en el primer franquismo». Hispania Sacra 70, 142: 681-689. https://doi.org/10.3989/hs.2018.047

$\begin{array}{ll}\text { Recibido/Received } & 25-01-2017 \\ \text { Aceptado/Accepted } & 25-01-2017\end{array}$

\section{Un ESTADO dE LA CUESTIÓN. Aspectos METOdológicos}

En la última década, los trabajos académicos sobre el papel de los católicos en la configuración política del franquismo han tomado actualidad. En este sentido, a las investigaciones llevadas a cabo por Feliciano Montero sobre el movimiento católico contemporáneo en España así como

\footnotetext{
${ }^{1}$ cbarreiro@ceu.es / ORCID iD: http://orcid.org/0000-00020121-9771
}

a los rigurosos trabajos de Cárcel Ortí, José Andrés Gallego, Javier Tusell o Sánchez Recio, convertidos en clásicos sobre la materia, tendríamos que añadir los estudios de los profesores Gonzalo Redondo, Manuel Álvarez Tardío, Roberto Villa García o Mónica Moreno Seco. En 2010 y a raíz de la conmemoración del centenario de la Asociación Católica Nacional de Propagandistas -entendida como núcleo aglutinador del catolicismo en respuesta a la necesidad de una presencia pública - se publicaron en CEU-Ediciones, los cuatro volúmenes de la Historia de la 
Asociación Católica de Propagandistas, con los que se retomó el interés por el análisis de la influencia de los católicos sociales en la coyuntura del siglo XX, que parecía haberse relegado desde que Mercedes Montero publicase en 1993 y 2001, en Eunsa, su visión de la asociación. ${ }^{2}$ Por todo ello, se ha dado pie al inicio de nuevas líneas de investigación que tratan de vehicular los principios fundacionales de los propagandistas con la política, las relaciones internacionales o la diplomacia, la prensa, el orden económico y cultural o incluso, la articulación de una oposición al franquismo desde el campo de la llamada «democracia orgánica». Los trabajos de los profesores Pablo Sánchez Garrido, Julio de la Cueva, Cristóbal Robles, Martín Puerta o Pablo Martín de Santa Olalla, tratan de cubrir un vacío importante en las aportaciones académicas realizadas sobre estas temáticas. ${ }^{3}$ También resultan reseñables, las tesis doctorales defendidas al respecto, que como las de Pablo Gutiérrez Carreras, Los hombres de la Asociación Católica de Propagandistas en la preparación de la Transición (CEU-San Pablo, 2016), Jesús Cogollos, El salario familiar en la actuación política de la CEDA durante el segundo bienio de la Segunda república (CEU-San Pablo, 2017), Juan José Malpica, El proyecto periodístico de Angel Herrera Oria: origen e implantación de la Escuela de Periodismo de la Iglesia y su influencia en la enseñanza (CEU-San Pablo, 2017), José Luis Orella, El origen del primer catolicismo social español (UNED, 2012) o la de Manuel Gutiérrez Navas, Biografía de Máximo Cuervo Radigales. Aproximación a una vida jurídico-militar y católico social (UNED, 2004) constituyen una avance sustancial de cara a la construcción de una necesaria panorámica científica sobre la influencia del pensamiento de los propagandistas católicos en la sociedad. Otros trabajos como los del prolífico Antonio Manuel Moral Roncal, Ana Campos Noguerol, o las del profesor Antonio César Moreno Cantano contribuyen de un modo más tangencial, a ofrecer una perspectiva también necesaria sobre la cuestión.

En este sentido, y en línea con las tendencias historiográficas esbozadas, en el presente artículo se trata de profundizar en la influencia de la ACNdeP en los primeros años del régimen: desde que Franco nombra su primer Gobierno en 1938, hasta que se forma el gabinete de 1945 en el que -terminada ya la Guerra Mundial- el papel de los propagandistas toma relevancia de la mano de hombres como Alberto Martín Artajo o José María FernándezLadreda. Esta investigación responde a la escased de análisis científicos, que se ha percibido respecto a la intervención de los propagandistas de Martín-Sánchez en este periodo inicial del franquismo, pues la mayor parte de las contribuciones al respecto, han mostrado su interés o bien hacia los simpatizantes de Falange y su papel en la articulación de un modelo político de corte totalitario (los recientes estudios del profesor de Diego González) o ya hacia la labor de los propagandistas en la etapa inmediatamente posterior, la fase central del régimen de Franco, cuando terminada la guerra europea los católicos se convierten en una cantera imprescindible de cara a solventar el claro problema de «aislamiento internacional». Por ello, y en cuanto que

\footnotetext{
2 Cárcel 2008; Gutiérrez, Barreiro y Martín Puerta 2010; Montero 1993 y 2001.

3 Barreiro Gordillo 2012; Martín de Santa Olalla 2003 y 2012; Martín Puerta 2015; Robles Muñoz 2009; Sánchez Garrido 2017.
}

desde la publicación del libro de Montero parece obviarse un campo de estudio que - a nuestro juicio- se computa como fundamental para gestar los pilares de la consolidación de los católicos propagandistas como familia preminente en el franquismo de las décadas de los 50 y 60 , se ha tratado de circunscribir este trabajo al tiempo en el que, desde un papel secundario y con recelo por parte de los elementos afines a Falange, los hombres de la ACNdeP fueron consquistando posiciones en las instituciones nacientes en puestos de diferente responsabilidad y naturaleza, aunque mostrándose idóneos para crear una administración estatal proclive a los intereses católicos y a su doctrina social. Para ello, se ha empleado una metodología descriptiva en la que se plasman los resultados de la consulta al Archivo de la ACNdeP, con cartas y documentos en los que se evidencia la influencia del catolicismo social ya en los años finales de la década de los cuarenta, y del Archivo Ángel Herrera, además de la bibliografía de referencia y la información proporcionada por el Boletín de la ACNdeP y la prensa vinculada a los propagandistas, teniendo muy presente el carácter específico de las aportaciones hemerorgáficas como fuente histórica. ${ }^{4}$

\section{LA ACNdeP en El CONTEXto eSPañol de LA Guerra Civil}

La guerra supuso muchas dificultades para la Asociación Católica Nacional de Propagandistas (ACNdeP) fundada por el jesuita Ángel Ayala en 1909 y que bajo la presidencia de Ángel Herrera, se había convertido en el principal elemento aglutinador del movimiento católico español. Impulsora de la CEDA, el partido confesional liderado por José María Gil Robles en la II República, y ferviente defensora de los principios de "accidentalidad» emanados de la doctrina de León XXIII, había sufrido los sinsabores del conflicto civil que enfrentaron a los españoles entre 1936 y 1939 y en el que la cuestión religiosa, se convirtió en un elemento capital: muchos centros de los propagandistas fueron saqueados y los socios - de variadas afiliaciones políticas - sufrieron represión. Presidida durante todo el periodo de guerra por Fernando Martín-Sánchez Juliá, los miembros de la Asociación fueron perseguidos en la zona republicana por su condición de católicos y por sus diferentes formas de concebir la acción pública.

Pero desde que Franco se hizo con las riendas del Estado, fueron muchos los que culparon a los propagandistas de haber mantenido una posición ambigua durante la República

\footnotetext{
4 Para el conocimiento interno de la Asociación, el Boletín de la $A C N d e P$ se convierte en fuente principal pues, aunque incurre en errores de citación y contenido, es una referencia de valor documental. En el Archivo de la ACNdeP, se han encontrado documentos interesantes para el cuerpo de este trabajo. Téngase en cuenta que este archivo se quemó en 1936 y que El Debate, comenzó su andadura en noviembre de 1911. El diario Ya, si reaparece después de la Guerra Civil igual que otros diarios vinculados a EDICA. La Junta Directiva de la ACNdeP al terminar la Guerra Civil era la siguiente: presidente, Fernando Martín-Sánchez y vicepresidente José Ignacio Isusi. Cuatro consejeros de nombramiento presidencial (Alberto Martín Artajo, Antonio Llombart, Alfredo López y Juan de Contreras, marqués de Lozoya) y cuatro consejeros nombrados por la Asamblea (José María de Peñaranda, Sabino Álvarez Guendín, Enrique Calabia y Luciano de Zubiría), un secretario general (José María Sagüés Irujo), un tesorero general (Juan Villalonga) y el consiliario nacional, Máximo Yurramendi.
} 
y no identificarse con las ideas falangistas, en boga en los años iniciales del régimen. Con todo, la ACNdeP se dispuso a continuar su obra de configuración católica del cuerpo social en convivencia con el nuevo orden político. Se iniciaba una etapa de lenta recuperación de afiliación, cuadros dirigentes y patrimonio, seriamente afectados por la guerra. La Asociación se manifestó a favor de los vencedores aunque como institución apostólica y estatutariamente "apolítica», dejó libertad a sus miembros para posicionarse a favor de unos $u$ otros postulados dentro de la España nacional. Sin embargo, los católicos españoles estaban bastante desorientados y entre los miembros de la ACNdeP se dieron ideas y opiniones confusas. En su conjunto los propagandistas contribuyeron a la institucionalización del régimen franquista y se mostraron partidarios de un Estado fuerte aunque distanciado de las ideologías de raíz pagana. Pero a pesar de la aceptación y «necesidad de cooperación» de la que siempre habló MartínSánchez, entre los miembros de la Asociación se abrió una falla que hizo que las discrepancias internas entre los católicos españoles saltasen a la esfera pública, especialmente después de que la Guerra Mundial Ilegara a su fin. ${ }^{5}$

\section{LOS PROPAGANDISTAS EN LAS ESTRUCTURAS DEL ESTADO}

En agosto de 1939, Franco formó su segundo Gobierno. Se trata de un gabinete de mayoría falangista en el que el propagandista José Larraz, ocupa el Ministerio de Hacienda y otro destacado católico, el acedepista y antiguo cedistas, José Ibáñez Martín, es designado ministro de Educación. El también propagandista Pedro Gamero del Castillo, fue nombrado ministro sin cartera y vicesecretario general del Movimiento. ${ }^{6}$ Ello llevó a que, aun teniendo presente esta distancia formal establecida con los sectores más proclives al falangismo y las particulares afinidades políticas de los militares también en el Gobierno, los propagandistas comenzaran a desempeñar un papel relevante de cara a la reestructuración del Estado nacional con una progresiva adhesión a las estructuras orgánicas oficiales, decisivas para la vertebración de un franquismo en ciernes. Así, observamos cómo desde los primeros momentos del régimen, miembros de la $\mathrm{ACNdeP}$ se fueron incorporando a los organismos dependientes del estado, en cargos de responsabilidad de diversa naturaleza y colaboraron también, con el poder civil a través de sus puestos como dirigentes de asociaciones católicas que se habían fraguado en años anteriores.

La documentación consultada en el Archivo de la ACNdeP, contrastada con las páginas del Boletín y un listado de propagandistas de elaboración propia, además

\footnotetext{
5 Barreiro Gordillo 2010: 73-74.

6 Ningún propagandista había formado parte del primer Gobierno de Franco que se nombró en Burgos el 30 de enero de 1938. Larraz, López Garrido y Velarde 2011. Pedro Gamero del Castillo, letrado del Consejo de Estado, representa una contradicción parecida a la de Serrano Suñer: ambos eran católicos militantes aunque defendían su pertenencia a Falange y posiciones totalitarias. Payne 1998: 353-517. Existe una copiosa bibliografía sobre los orígenes del Estado nacional. Puede verse; Orella Martínez 2001. Juan Beigbeder, Ramón Serrano Suñer, José Varela, Salvador Moreno Fernández, Juan Yagüe, Esteban Bilbao, Luis Alarcón de Lastra, Joaquín Benjumea, Alfonso Peña Boeuf, Rafael Sánchez Mazas y Agustín Muñoz Grandes, ocupaban el resto de las carteras ministeriales.
}

de la bibliografía de referencia que existe sobre la materia, nos ha permitido comprobar cómo los propagandistas se integraron en el nuevo estado mediante el desempeño de responsabilidades políticas en las Cortes, los Gobiernos civiles, ayuntamientos y corporaciones locales, institutos regionales y organismos de carácter político-cultural. Los propagandistas además, ocuparon diferentes puestos como asesores técnicos de organizaciones públicas y fueron partífices en la elaboración, intervención y redacción de la nueva legislación con la que se estaba cimentando el Estado. En estos años, su presencia fue especialmente relevante en las instituciones dependientes de los Ministerios de Educación y Justicia. En este sentido, los materiales documentales a los que se ha tenido acceso, permiten elaborar la siguiente relación de miembros de la ACNdeP que formaron parte de la administración del régimen entre 1939 y $1945 .^{7}$

Como subsecretarios o directores generales, encontramos a los siguientes miembros de la ACNdeP: Enrique Calabia, subsecretario de Hacienda; Luis Montes director general de Telégrafos; C. de Argillo, de Minas; Francisco Boch Marín, de Sanidad -era subdirector general aunque director en funciones-; Joaquín Espinosa, de Beneficencia (como el anterior); Ignacio de Casso y Romero, director general de Registros y Notariados $-\mathrm{y}$ desde 1941, director general del CEU—; Máximo Cuervo Radigales, de Prisiones, Mariano Puigdollers, de Asuntos Eclesiásticos; Marqués de Lozolla, director general de Bellas Artes; González Barrio, de Propiedades; Luis Ortiz, de Enseñanza Media; Enrique Jiménez Arnau, director general de Prensa y Pérez de Ayala, de Trabajo. ${ }^{8}$ En puestos de gobernadores civiles, aparecen los propagandistas Juan Antonio Cremades, de Lérida, José Clavero, de Tenerife hasta 1943 y en delante de Segovia, Luis Martín Ballesteros, de Logroño y Ávila (ya en 1946) y Francisco Sanz de Tejada, barón de Benasque, de Zaragoza y desde 1944 de Guipúzcoa. Y en las corporaciones municipales o ayuntamientos tenemos alcaldes como Rafael Bonet, Teruel (1941) y Vicente Lobo, Palencia (1942), hasta puestos como el de Rigoberto Albor Vicens, teniente de alcalde del ayuntamiento de Alcoy en 1941; Joaquín Ruiz-Giménez, primero concejal del ayuntamiento de Madrid con Alberto Alcocer como alcalde; Ignacio Arango, teniente de alcalde del ayuntamiento de Gijón en 1943; Rafael Bittini, concejal y teniente de alcalde de Las Palmas, en 1943 y Valentín Fernández Badía, teniente de alcalde de León en 1942. Como procuradores en Cortes, nos encontramos a Luis Ortiz Muñoz (1943) y al marqués de la Vega de Anzó (1943) y durante los años siguientes, los propagandistas Fernando María Castiella - primero director del Instituto de Estudios Políticos y más adelante, decano de la Facultad de Ciencias Políticas y Empresariales - Antonio Mateos, Mariano Puigdollers, Antonio Blasco del Cacho, José Larraz y Julián Laguna Serrano, también van a ser designados procuradores.

Durante estos primeros años de formación del Estado franquista y también dentro de las estructuras oficiales,

\footnotetext{
7 Archivo ACNdeP. Carta de Gregorio Santiago Castiella, secretario general del Consejo Superior de Protección de Menores (27 enero 1945). Se ha revisado, ampliado y completado, la relación publicada en Barreiro Gordillo 2010: 88-93.

8 Archivo ACNdeP. Carta de Gregorio Santiago Castiella, secretario general del Consejo Superior de Protección de Menores (27 enero 1945).
} 
nos encontramos con otros propagandistas destacados, como lo fueron Antonio Reverte Moreno, director de La Verdad y diputado cedista por Murcia, quien en 1940 es nombrado encargado de prensa extranjera en el Ministerio de Gobernación y que años después, ocupará importantes puestos en las instituciones políticas y económicas de su región. ${ }^{9}$ También lo fue, Jesús Rodríguez García de los Salmones, secretario del Instituto de Moneda Extranjera así como el secretario de prensa del Ministerio de Asuntos Exteriores, José Luis García Rubio. Más conocida es, por otra parte, la trayectoria profesional de los periodistas y propagandistas, Vicente Gallego Castro y Pedro Gómez Aparicio, director y subdirector de la Agencia EFE, respectivamente. ${ }^{10}$ Pero, entre los miembros de la ACNdeP que participaron en la política de estos años también hemos localizado asesores técnicos, directivos de los diferentes Consejos Provinciales de FET de las JONS y miembros de la Diputación. Todos ellos contribuyeron desde sus cargos, a la consolidación del Estado y se integraron en las nuevas instituciones.

Pero el Ministerio de Educación Nacional fue la parcela de mayor influencia de los propagandistas, al estar a cargo de José Ibáñez Martín, exdiputado de la CEDA por Murcia y ministro durante doce años (1939-1951). Será además, presidente del Consejo Superior de Investigaciones Científicas (CSIC, 1939-1943). De las seis Direcciones Generales en las que se dividía el ministerio, dos de ellas van a estar ocupadas por personalidades de la ACNdeP; la Dirección General de Enseñanza Media, en manos del antiguo articulista de El Debate, Luis Ortiz Muñoz quien en escasos meses había ocupado ya los cargos de consejero nacional de Cultura, secretario del Servicio Español de Profesorado, secretario técnico del Ministerio de Educación Nacional y secretario general del Consejo Nacional de Educación; y la Dirección General de Bellas Artes, ocupada por el consejero de la ACNdeP y diputado cedista, Juan de Contreras y López de Ayala, marqués de Lozoya, que se mantendrá en el cargo hasta 1951. En 1942 el propagandista del Centro de Madrid, José Pemartín, va a ser nombrado director general de Enseñanza Superior. También propagandistas eran el asesor técnico de la Dirección General de Primera Enseñanza, Alfonso Iniesta; el inspector jefe de Primera Enseñanza, Mariano Santos y el miembro del Consejo Nacional de Educación, Isidoro Martín, antiguo secretario del Centro de Madrid. Por otra parte, durante estos primeros años del régimen, los propagandistas Miguel Sancho Izquierdo, Enrique Luño y Sabino Álvarez Gendín, serán nombrados respectivamente rectores de la Universidad de Zaragoza, Barcelona y Oviedo.

Dentro del Ministerio de Educación Nacional y por Ley de 24 de noviembre de 1939, se había creado el Consejo Superior de Investigaciones Científicas (CSIC), organismo nacido para orientar, estimular y coordinar la investigación científica en España. El presidente, era el ministro Ibáñez Martín y el secretario general, el todavía propagandista José María Albareda. Rafael de Balbín y Lucas, fue nombrado vicesecretario general de Publicaciones y encargado de la revista oficial de la

\footnotetext{
9 Pérez Crespo 2013.

10 Sobre la trayectoria de estos periodistas en los años objeto de este estudio puede verse Pizarroso Quintero 2009 y Martín Aguado y Vilamor 2012.
}

institución, Arbor. Manuel Bermejillo, del Centro de Madrid, era otro de los integrantes de esta institución. ${ }^{11}$ Juan Manzano, Pedro Rocamora, Francisco Cantera y Jaime Guasp Delgado, también colaboraron en la labor desempeñada por el CSIC en estos primeros años de la década.

El Ministerio de Justicia va a ser otra plaza fuerte de participación de los propagandistas en el estado franquista ya que, aunque controlado por el tradicionalista Esteban Bilbao, este ministerio se convierte en un núcleo principal para la colaboración política de muchos católicos a través de sus diferentes brazos e instituciones. En este sentido y de manera especialmente activa, fue la participación de los propagandistas en el Patronato de Redención de Penas por el Trabajo así como los diferentes tribunales o juntas de protección de menores y de la mujer. El Patronato de Redención de Penas por el Trabajo, estaba presidido por el propagandista Máximo Cuervo Radigales, director general de Prisiones, y había sido creado por orden del Ministerio de Justicia en octubre de 1938 . $^{12}$ Entre los propagandistas que participaron en él, además de su presidente Máximo Cuervo, encontramos a Vicente Jordá Botella, del Centro de Alcoy que en 1941 es el presidente de su Junta local; Antonio Lázaro Sapiña, del Centro de Valencia y miembro del Patronato de Redención de Penas por el Trabajo, que en 1944 creará en Valencia la "Delegación local pro-presos» así como al propio Fernando Martín-Sánchez, jefe de las Juntas locales para la Redención de Penas por el Trabajo desde 1945. El Tribunal y las diferentes Juntas de Protección de Menores, estaban también adscritos al Ministerio de Justicia, y desde ellos los propagandistas desempeñaron un papel interesante. Así, encontramos a los siguientes miembros de la ACNdeP con responsabilidades a nivel nacional y local: Mariano Puigdollers, en 1941 presidente del Tribunal de Apelación de Menores y vicepresidente del Consejo Superior de Protección de Menores; José Rodríguez Soler, vocal en 1941 de la Junta provincial de Protección de Menores; Francisco Manich, secretario general de la Protección de Menores en 1944; el conde de Trigona en 1941 vocal de la Junta provincial de Protección de Menores y luego presidente de la Junta de Protección de Menores hasta 1947, año en el que dimite por motivos de salud; Gregorio Santiago Castiella, en abril de 1941 vocal del Tribunal de Apelación de Menores hasta que en 1944 sea designado secretario general del Consejo Superior de Protección de Menores; Mariano Díaz Plaja, en mayo del 42 vocal de la Junta provincial de Protección de Menores de Toledo; José Fernández Borrajo, 1941 presidente del Tribunal Tutelar de Menores de Orense desde 1940; Antonio Ferrer Sama, presidente del Tribunal Tutelar de Menores de Murcia desde 1941; José Pérez Ávila, secretario de la Junta provincial de Protección de Menores de Orense en 1942 y Francisco Siso Cavero, secretario de la Junta provincial de Protección de Menores de Murcia desde 1945. Por su parte, en el Patronato de Protección a la Mujer participaban

11 El CSIC estaba dividido en patronatos - Raimundo Lulio, Menéndez y Pelayo, Alfonso El Sabio, Cierva, Ramón y Cajal y Alonso Herrera - y cada uno de ellos tenía dentro de sí una serie de institutos. Había además Delegaciones Provinciales en las que encontramos a destacados propagandistas locales. Sobre Arbor, véase: Arbor, n-s 479-480. Monográfico «40 años de Arbor: un análisis autocrítico». Nov-dic. 1985.

12 Gutiérrez Navas 2004. 
los siguientes miembros de la Asociación: Alberto Martín Artajo, como vicepresidente; Julián Pascual Dodero, su secretario general del Patronato de Protección a la Mujer desde 1942 y hasta su sustitución en 1944 por José María Sánchez de Muniain. Como vocales, fueron miembros de este organismo, Manuel Bermejillo, Mariano Puigdollers, Carlos de la Mora Pajares, José María de Urquijo, José María Sánchez de Muniáin Gil, Arsenio González de la Vega y Gregorio Santiago Castiella.

\section{LOS PROPAGANDISTAS Y SU PAPEL MEDIADOR EN LAS RELACIONES IGLESIA/ESTADO}

Resulta interesante tener presente cómo conforme las influencias totalitarias ganaron poder político en el Estado durante el primer franquismo, la Iglesia establecía un distanciamiento con las nuevas directrices: el 8 de agosto de 1939 el cardenal primado Gomá -coordinador de la Carta Colectiva - firmó la pastoral «Lecciones de la guerra y deberes de la paz» en la que aludía al deber de los católicos de participar en la acción política aunque mostrándose reticente con las posiciones gubernamentales. ${ }^{13}$ Cuando el Estado español censuró la encíclica de Pío XII "Summi Pontificatus», que contenía párrafos poco favorables para Alemania y a las consecuencias nefastas de la autonomía del Estado, el nuncio Gaetano Cicognani, _embajador de la Santa Sede ante las autoridades políticas nacionales- llevó a cabo las gestiones oportunas para su publicación. Y fue precisamente la ACNdeP, quien se hizo cargo de la primera edición íntegra que se hizo en España, de esta encíclica. ${ }^{14}$

Sin embargo, es cierto que a pesar de la influencia que se ejercía en el Gobierno por parte de algunos elementos de Falange, las autoridades deseaban una España católica. En este sentido, la Iglesia vivió un momento óptimo en cuanto gozó de protección legal; durante los años de guerra, la España de Franco había desmantelado la legislación republicana y ahora tenía que reconstruir un Estado que fuera positivamente católico. ${ }^{15}$ Así, la legislación que se aprobó a partir de 1940 se orientó en esta dirección: se restablecieron las cátedras de Historia de la Iglesia y de Derecho Canónico, se derogó la Ley de divorcio o se procedió a la anulación de los matrimonios civiles efectuados hasta entonces. ${ }^{16}$ Pero pese a ello, las relaciones entre el

13 Véase Martín de Santa Olalla 2003. También, Redondo 1999 y Ruiz Rico 1977. El organigrama de la Jerarquía Eclesiástica en España era el siguiente: papa, Pío XII (1939-1958); secretarios de Estado - ministro de Exteriores de la Santa Sede- Maglione (marzo 1939-agosto 1944); Nunciatura -embajada de la Santa Sede ante las autoridades políticas nacionales- Gaetano Cicognani (16 mayo 1938-octubre 1953); Episcopado nacional. Preeminencia del arzobispo-primado de Toledo. Isidro Gomá (muere en 1940) y Pla y Deniel (octubre 1941 a julio 1968); Embajadores de España ante la Santa Sede, José Yanguas Mesía (julio 1938-octubre 1942) y Domingo de las Bárcenas (octubre 1942-1946).

14 Barreiro Gordillo 2010: 200.

15 Payne 1984: 231.

16 El 23 de octubre de 1939, se aprobaba la Ley de restablecimiento del Presupuesto de Culto y Clero que mejoraba la situación económica de la Iglesia. Se destinarían 2.600 .000 pesetas para la enseñanza y el profesorado en los seminarios españoles, lo cual significaba un aumento de un millón sobre lo que figuraba en el último presupuesto anterior a la República. Del mismo modo, para la conservación ordinaria de iglesias, es decir, para los gastos corrientes de culto y obras se aumentaba la partida hasta 1.600 .000 pesetas. La reconstrucción de iglesias destruidas no estaba
Gobierno y la Jerarquía no terminaban de ser cordiales: la Santa Sede no estaba dispuesta a que Franco interfiriese en las competencias de la Iglesia ni a verlas supeditadas a su poder político. Así las cosas, en junio de 1941, se firmó el acuerdo entre la Santa Sede y el Estado español en virtud del cual, el Gobierno se comprometía a no legislar sobre materias mixtas o sobre aquellas que pudieran interesar a la Iglesia sin previa negociación con la Santa Sede. Ambas potestades se urgían además, a concluir lo antes posible un nuevo concordato que sustituyera al de 1851 , pero mientras tanto, los cuatro primeros artículos de este se mantendrían en vigor: el primero reconocía la religión católica como única de la nación y los otros tres, se referían a la inmunidad jurídica del clero y al reconocimiento de un cierto derecho de control de la Iglesia sobre la enseñanza, la prensa y las publicaciones. En cuanto al derecho de presentación de obispos, no se ejercería de la forma directa hasta 1931: en adelante el Gobierno negociaría con la Nunciatura cada nombramiento, propondría sobre esa base seis nombres ( «la seisena»), de los cuales el papa escogería a tres («la terna») y Franco presentaría uno de ellos que sería nombrado por el pontífice. ${ }^{17}$ La estabilización del franquismo y el enorme número de diócesis que se hallaban sin obispo, fueron las causas que indujeron a Pío XII a dar este paso en la búsqueda de la concordia con el Gobierno de Franco. El acuerdo de 1941 limó las tensiones, puso de manifiesto una voluntad de concordia y aunque el tono fue más cordial y confiado, tampoco faltaron contratiempos.

Tras la firma de los acuerdos de 1941 entre el Gobierno y el Vaticano, se consolidaron las relaciones entre el Estado y la Iglesia, pasándose, en palabras de profesor Andrés Gallego, de cierto laicismo a "franca colaboración». ${ }^{18}$ Pero será a partir de febrero de 1943 y a raíz de la aprobación de la Ley de Cortes de 1942, cuando la Jerarquía Eclesiástica entre de lleno en el aparato del régimen. Fernando Martín-Sánchez en calidad de presidente de la ACNdeP, felicitó a los arzobispos de Toledo, Granada y Burgos y a los obispos de León y Barcelona, por su nombramiento como procuradores a Cortes. ${ }^{19}$ Desde 1943 existió una "voluntad real» de construir un Estado católico dejando atrás los enfrentamientos de 1939-1940, cuando desde la prensa - regida por los falangistas de la Dirección de Propaganda - se insistía en la necesidad de restablecer el Concordato de 1851 con su carga regalista. Además y en línea con este restablecimiento de la cordialidad entre ambas instituciones, por Circular de 25 de marzo de 1944, se eliminaba la censura a las publicaciones eclesiásticas. En octubre de 1944, se creaba, dependiente del Ministerio del Interior, la Subsecretaria de Asuntos Eclesiásticos, se reorganizaba el cuerpo de capellanes de prisiones y el de

incluida en estas consignaciones, porque se cargaba a los presupuestos de regiones y edificios devastados. Boletín ACNdeP (1 noviembre 1939).

17 Vid. Andrés Gallego y Pazos 1999 y Martín de Santa Olalla 2003: 53-72.

18 Andrés Gallego y Pazos 1999: 67.

19 Archivo ACNdeP. Correspondencia de Fernando Martín-Sánchez. La circular dice: «En nombre de la Asociación, en el del C.E.U. y en el mío propio, tengo el honor de elevar a V.E. mi felicitación por haber sido nombrado Procurador en Cortes por el Caudillo. Esperando grandes bienes para la Iglesia y para la Patria de su presencia en las Cortes, encomienda a sus oraciones la Asociación, el C.E.U. y su modesta persona y besa humildemente su Pastoral Anillo». 
asesores eclesiásticos del Sindicato Único. En pocos meses, las relaciones entre Iglesia y Estado fueron mejorando hasta terminar convergiendo en el "nacionalcatolicismo» característico de la década de los 50 y en el que tanto tuvieron que ver los hombres de la ACNdeP.

LOS PROPAGANDISTAS Y LA POLÍTICA EDUCATIVA: LA LEY DE ORDENACIÓN UNIVERSITARIA (1943) Y LA LEY DE EDUCACIÓN PRIMARIA (1945)

Los propagandistas se mostraron satisfechos con la legislación aprobada por el Gobierno a partir de 1943. Y no solo con las disposiciones directamente relacionadas con las potestades de la Iglesia, sino también con aquellas que de una manera menos directa, pudiesen contribuir a crear un marco confesional de "socialización política»: aquello que fuese más allá del simple diálogo Iglesia/Estado. ${ }^{20}$ Aunque la ACNdeP como entidad corporativa no se manifestó de manera pública respecto a la política, lo cierto es que los propagandistas cooperaron, participaron y agradecieron muchas de las disposiciones emanadas del Gobierno y especialmente aquellas que afectaban a la educación.

En los primeros años 40, la actividad de Ibáñez Martín al frente del Ministerio de Educación Nacional no siempre fue bien vista en el seno de sus compañeros propagandistas, en cuanto en ocasiones toleró tendencias próximas al estatismo imperante. No obstante, a este respecto ya se ha visto cómo desde las primeras semanas de la Guerra Civil, se habían adoptado disposiciones que primaban los intereses del catolicismo: en educación se había restaurado la enseñanza religiosa en las escuelas no confesionales y en virtud de una ordenanza de la Junta de Defensa Nacional de fecha de 22 de septiembre de 1936, se había restablecido la influencia de la doctrina católica cobre el conjunto de la enseñanza. En esta misma línea, la legislación de los años 40 confirma el carácter obligatorio y general de la enseñanza religiosa y la extiende a los tres ciclos de enseñanza: primaria, secundaria y superior. El 29 de julio de 1943 -con Ibáñez Martín al frente del ministerio- era promulgada la Ley de Ordenación Universitaria por la que se pretendía afrontar la tarea de "cristianizar la vida de la Universidad» del Estado en la idea de crear unas universidades educativas que atendiesen tanto el conocimiento de lo religioso y político como de la educación disciplinada de los estudiantes. ${ }^{21}$ En la elaboración del proyecto tomó parte el propagandista del Centro de Oviedo, Sabino Álvarez Gendín - rector de la Universidad de Oviedo, miembro del Consejo de Rectores y procurador a Cortes españolas - aunque su aprobación final fue controvertida. En este sentido, el también propagandista Isidoro Martín fue quien durante la celebración de la XXIX Asamblea General de la ACNdeP, la del año 42, apuntó a la problemática que se cernía entorno a la aprobación de la reforma planeada y a las dificultades que se estaban encontrando las autoridades ministeriales ${ }^{22}$ :

20 Hermet 1985-1986: vol. I, 180.

21 Respecto a la Educación y Universidad durante los años 40 en España pueden verse las obras: Alted Vigil 1991: 95-124; Mayordomo Pérez 1999; Peset 1991.

22 Véase Laín Entralgo 2004: 292-293, donde el autor —en su condición de consejero nacional del Movimiento y procurador a Corteshace referencia a las negociaciones entorno a la aprobación de la Ley
[...] el espíritu que informa es un espíritu fundamentalmente católico; es decir, sus promotores más altos están animados del mejor deseo de servir fidelísimamente a la Iglesia católica. Sin embargo, no nos hagamos ilusiones. Estos altos promotores de la Reforma tropiezan, me refiero ciertamente a la Ley de Universidades, con alguna oposición entre personas que ocupan tan elevadas posiciones. Por ejemplo, una de esas personas decía: "Solo hay un punto espinoso: el de la Universidad que no sea mantenida por el Estado. El derecho a enseñar es un derecho que compete al Estado y no cabe hablar de participaciones». De modo que lo digo también pensando en la actuación de los que quieren servir generosamente a la Iglesia. ${ }^{23}$

Parece que estamos asistiendo a una confrontación entre dos maneras diferentes de entender la participación de los propagandistas católicos en la política, pues probablemente Isidoro Martín estuviese haciendo referencia a hombres que como Laín o Tovar, defendían las posiciones de Falange al considerar que el derecho a enseñar competía al Estado. Pero para los propagandistas además, el tema de la cultura religiosa en las Universidades debía de ser una cuestión a plantear en un futuro plan general de enseñanza. El propio arzobispo de Toledo presentó una enmienda al proyecto de ley que fue aprobada en el articulado final y que estableció cómo «el Estado español reconoce a la Iglesia en materia universitaria sus derechos docentes, conforme a los cánones y a lo que en su día se determine mediante acuerdo entre ambas partes». En el texto definitivo, art. 3ㅇ se establece que «la Universidad, inspirándose en el sentido católico consustancial con la tradición universitaria española, ajustará sus enseñanzas a las del dogma y la moral católica, como también a las normas del derecho canónico vigente», es decir, se establecía la confesionalidad católica de la Universidad y en el artículo 6 se añade que «la Universidad se coloca bajo la advocación y el patronato de Santo Tomás de Aquino, cuyo día será festivo y solemnizado con los actos religiosos y académicos».

Por otra parte, esta Ley de Ordenación Universitaria de 1943, suprimía la enseñanza libre, por lo que la asistencia a las clases de la Universidad empezó a ser obligatoria para todos los alumnos. Quedaban dispensados de tal escolaridad los alumnos que cursaran sus estudios en los centros de enseñanza universitaria del Sacromonte de Granada, de María Cristina de El Escorial y Deusto. Dos años más tarde, una Orden ministerial de 11 de julio de 1945 reconocía al CEU (iniciativa de los propagandistas desde su creación) como centro adscrito a la Universidad de Madrid. Con ello sus alumnos quedan también dispensados de asistir a las clases de la Universidad y el CEU, equiparado a los tres centros privados mencionados. ${ }^{24}$

En enero de 1944, adquirió una importancia relevante la aprobación del Decreto relativo a la Enseñanza Religiosa en las Universidades, ampliación de los preceptos que figuraban ya en la Ley de Ordenación Universitaria y que cuenta con el beneplácito de Martín-Sánchez y de Martín Artajo, por

de Ordenación Universitaria y la posición que como presidente de la Comisión desempeñó Leopoldo Eijo en pro de «los derechos de la Iglesia».

23 Boletín de la ACNdeP (15 octubre 1942) p. 7.

24 Barreiro 2010: 195. 
entonces secretario del Consejo de Estado. ${ }^{25}$ Este Decreto, prescribía una hora semanal de enseñanza sobre materias religiosas en la Universidad lo que representaba, cerca de treinta y cinco horas en cada curso escolar. La vida religiosa universitaria estaría sometida a un sacerdote nombrado por el prelado respectivo y confirmado por el rector y el Ministro de Educación Nacional. Una década después, en el Concordato de 1953 se consagrarán definitivamente estos principios.

En julio de 1945 se aprobó una nueva Ley de Educación Primaria, patrocinada también por José Ibáñez Martín y que afectaba a los intereses del catolicismo oficial. "No hay Código, ni Concordato, ni legislación escolar que aventaje a nuestra Ley de Enseñanza Primaria por su fidelidad a la doctrina católica», parece que dijo Ibáñez Martín. El ministro agradeció a la Comisión de Prelados su colaboración con la redacción de la ley y en especial, al obispo de Madrid-Alcalá, de quien alabó «su admirable celo apostólico» y "su vivo y fecundo fervor español»; se declaró «consagrado enteramente a imprimir el más puro sentido cristiano en todas las manifestaciones de la enseñanza» con vistas a convertir a España en "columna inconmovible de la eterna religiosidad de Occidente» y justificó este propósito político, no como producto de una actitud personal o de "familia» en el seno del régimen, sino de la verdadera esencia del mismo. ${ }^{26}$ Como se ve, la imbricación entre los poderes eclesiásticos y civiles de los hombres de la ACNdeP en el tema de la enseñanza, llega a ser muy estrecho. Con la aprobación de la Ley de 17 de julio de 1945 sobre la Enseñanza Primaria - y ya en febrero de 1953, de la controvertida Ley de Enseñanza Media, en la que tan destacado será el papel del propagandista Joaquín Ruiz-Giménez y su equipo de Gobierno-, la influencia de la Iglesia se hizo palpable en todos los ciclos de la educación.

\section{EL MOVIMIENTO CATÓLICO ESPAÑOL COMO VALEDOR ANTE EUROPA}

Como consecuencia del cambio en el curso de la Guerra Mundial tras la batalla de Stalingrado, España debía de romper vínculos con el Eje y tratar de vender un modelo más acorde con las nuevas circunstancias. Franco se replanteó la idea de mostrar una imagen de apertura al exterior al hacer entrar en el Gobierno a elementos destacados del sector confesional católico, bien relacionados con las corrientes democristianas imperantes en Europa. Entre ellos, había

25 Archivo ACNdeP. Correspondencia de Fernando Martín-Sánchez. Carta de Martín-Sánchez a Rigoberto Domenech y Valls, arzobispo de Zaragoza, en la que le explica el contenido del Decreto de Enseñanza Religiosa en la Universidad. Es posible que en su aprobación existieran tensiones con la Federación de Amigos de la Enseñanza, alarmados porque en el Decreto «los actos de Culto y Devoción en el interior de la Universidad quedaban sometidos al representante universitario del Prelado de la Diócesis, sin intervención de otras Asociaciones o Congregaciones Religiosas». El 19 de julio de 1944, el Gobierno aprobaba además la Ley de Protección escolar, dirigida a los centros docentes de índole privada para que primasen una política de becas que beneficiase a los estudiantes brillantes con menos recursos económicos y así «no se malogre ningún talento por falta de medios económicos» y de que «todos los que lo merezcan tengan fácil acceso a los estudios superiores». Parece que a esta Ley respondió la política de becas que empezó a aplicarse en el CEU a partir de 1944.

26 Tusell 1984. muchos miembros de la ACNdeP que llevaban, al menos desde 1942, intentando estructurar un programa de unidad política basado en un sistema capaz de dar continuidad al régimen español. Los propagandistas en su conjunto, intentaron ofrecer una imagen corporativa de España, una muestra que eliminase todo resto de totalitarismo y representara en Europa el peso del catolicismo al servicio de Pío XII. Los propagandistas se habían servido de los Círculos de Estudios para criticar las teorías totalitarias e incluso intentaron buscar un punto común de pensamiento, que englobase la defensa corporativa que hacían del Estado. En esa línea divulgativa se había producido en la primavera de 1943, el viaje a Roma del propagandista Joaquín Ruiz-Giménez, presidente del organismo internacional de universitarios católicos Pax Romana, en un peregrinaje que contó con el beneplácito y la ayuda económica del Gobierno español. ${ }^{27}$ Los propagandistas, podrían convertirse así en los principales valedores de Franco en Europa ya que el régimen, se esforzaba ahora en ofrecer una imagen de apertura para mitigar el aislamiento de que era objeto por parte de las potencias vencedoras. En esta línea, dos de la Leyes Fundamentales del Estado, el Fuero de los Españoles aprobado el 17 de julio de 1945 -en cuya redacción tendrá un papel muy activo el propagandistas y futuro embajador ante la Santa Sede y ministro, Joaquín RuizGiménez-y la Ley de Referéndum Nacional de 22 de octubre de 1945, deben de entenderse en esta dirección: un intento por limar los recelos que desde exterior se sentían hacía el estado español.

Ante la condena internacional, el régimen apuesta por los católicos y la Iglesia lo hace por el régimen: el 9 de mayo de 1945 - antes de la formación del Gobierno- el cardenal Pla y Deniel hace pública una pastoral en defensa del régimen y el 28 de agosto, respalda el Fuero de los Españoles; el 18 de noviembre el papa Pío XII envía un elocuente mensaje al pueblo español y en diciembre, Joaquín Ruiz-Giménez visita Londres y Nueva York. ${ }^{28}$ Se trataba de movilizar a las juventudes, a los intelectuales y a las jerarquías católicas a favor de España. La celebración del XIX Congreso Mundial de Pax Romana en Salamanca y El Escorial, en julio de 1946, con la asistencia de más de trescientos delegados procedentes de treinta y dos países, se convierte así, en un primer paso hacia la consagración del papel de los católicos como mediadores del régimen de España ante Europa. Porque las

27 Ruiz-Giménez se entrevistó con el cardenal Pizzardo y con Pío XII. Según cuenta el protagonista en el Boletín de la ACNdeP (1 junio 1943), el pontífice se refirió con alabanza a la orientación del Estado español: «[...] llevó la conversación al terreno de España y nos indicó que la Iglesia miraba con gran simpatía la actitud de nuestra Patria, defendiendo los intereses de la Iglesia y de la Cristiandad en las conversaciones y en los conciertos europeos». Como síntesis al papel de Joaquín Ruiz-Giménez en los años previos a su nombramiento como ministro puede verse Muñoz Soro 2006: 259-288. Sobre este tema, pueden consultarse las diferentes aportaciones que encontramos en Sánchez Recio 2005.

28 Barreiro Gordillo 2012: 232-233. El cardenal Pla y Deniel publicaba el 1 de septiembre de 1945 una pastoral donde contraponía la Guerra Mundial que acababa de finalizar, con la "verdadera cruzada» que había sido la Guerra Civil española, destacaba el refrendo popular del Estado franquista, su catolicismo y «la orientación de cristiana libertad, opuesta a un totalitarismo estatista, del Fuero de los Españoles». Aunque -y aquí venía el apoyo directo a Martín Artajo- el primado reiteraba su aspiración a una liquidación rápida de la represión y a la urgente estructuración del Estado con participación ciudadana en el Gobierno y apertura de cauces a la opinión. 
circunstancias de aislamiento, obligaron a Franco a jugar abiertamente la carta católica, a buscar la «cobertura» de la Iglesia: una Iglesia que aunque se mostraba partidaria del régimen - Pío XII mantuvo al nuncio en Madrid, igual que los embajadores de Portugal y Argentina- había mantenido roces con Franco en materias como la unidad sindical o el nombramiento de los obispos. ${ }^{29}$ Pero ahora Franco encuentra en los hombres de la Asociación, una cantera imprescindible de cara a solventar el claro problema de "aislamiento internacional» que aparece a partir del final de la Segunda Guerra Mundial. Se trataba sencillamente de «reposicionar» internacionalmente a España y para ello el régimen utilizará la única carta con la que contaba: la de los políticos católicos de la ACNdeP que venían desde un lustro atrás, acoplándose en las instituciones del Estado para configurar el nuevo orden social.

El 21 de julio de 1945, se publicaba en la prensa la formación del nuevo gabinete que abría una nueva etapa en la historia de la ACNdeP. ${ }^{30}$ Dos propagandistas, Alberto Martín Artajo - nuevo ministro de Asuntos Exteriores- y el general de Artillería y ex-diputado cedista, José María FernándezLadreda -Obras Públicas- accedían por primera vez a carteras ministeriales. ${ }^{31}$ La vicesecretaria de Educación Popular fue adscrita al Ministerio de Educación, que continuó en manos del propagandista José Ibáñez Martín. Esta vicesecretaría tenía a su cargo el control de la prensa, la censura y la propaganda - que hasta entonces había estado encomendada a FET de las JONS - y que asumieron en 1946 los propagandistas Luís Ortíz Muñoz, como subsecretario de Educación Popular, Tomás Cerro Corrochano - por aquellos días una voz de "tendencia obrerista» dentro de la Asociación-, en el puesto de director general de prensa, y Pedro Rocamora y Valls como director general de propagan-

29 Terminada la Guerra Mundial la opinión internacional se vuelca en contra del régimen de Franco, al que considera dictatorial y carente de legitimidad democrática. El 12 de diciembre de 1946, la Asamblea de las Naciones Unidas votaba por 34 votos contra 6 y 13 abstenciones una moción que condenaba al régimen español y recomendaba la ruptura de relaciones con él (4 agosto 1945). Carta de Sagués a MartínSánchez sobre el nuevo Gobierno y los nuevos cargos y sobre el acuerdo de Postdam. Archivo ACNdeP. Correspondencia de Fernando MartínSánchez. En marzo de 1946 el cardenal norteamericano Spellman, máxima autoridad eclesiástica de su país, visitaba España. Para conocer la situación internacional de España desde el final de la II Guerra Mundial puede verse Portero 1989 y Martín Artajo 1950.

30 En la composición del Gobierno era palpable el declive de Falange ya que solo se mantenían Girón en Trabajo y Fernández Cuesta en Justicia. El gabinete, quedó integrado del siguiente modo: Gobernación: Blas Pérez González; Ejército: Fidel Dávila; Marina: Francisco Regalado; Aire: Eduardo González; Asuntos Exteriores: Alberto Martín Artajo; Hacienda: Joaquín Benjumea; Industria y Comercio: Juan Antonio Suánces; Agricultura: Carlos Rein; Justicia: Raimundo Fernández-Cuesta; Educación: José Ibáñez Martín; Obras Públicas: José María FernándezLadreda y Trabajo: José Antonio Girón de Velasco. Para conocer la evolución política del régimen de Franco desde la entrada de destacados propagandistas en el Gobierno es fundamental la obra Tusell 1984. También resulta útil Payne 1987 considerada como una de las mejores historias generales del régimen; Aguilar y Gil Pecharromán 2008; Portero 1989.

31 El Boletín de la ACNdeP destaca en primera página los nombramientos de Martín Artajo y Fernández Ladreda, y recoge los méritos que habían realizado como integrantes del Movimiento Católico. El Boletín hace también mención de la elevación del también propagandista Alfredo López, a la presidencia interina de la Junta Nacional de la Acción Católica Española. da. Esta transferencia de competencias acarreó la salida de Gabriel Arias Salgado y de Juan Aparicio, como subsecretario y director general, respectivamente. Todos estos nuevos nombramientos servían para potencian la colaboración del catolicismo político y marcan el inicio de la definición del régimen como un Estado católico. Desde este momento, la influencia de los hombres de la Asociación en la política será máxima: desde ministerios, organismos, instituciones públicas, institutos nacionales o ayuntamientos, muchos propagandistas continuaran contribuyendo a la vertebración/ desarrollo del régimen franquista y al establecimiento del catolicismo en la sociedad.

\section{CONCLUSIONES}

A partir de 1945, Franco encontró en los hombres de la ACNdeP la garantía necesaria para poner en valor su régimen ante el mundo y frenar el aislamiento político en el que se vió involucrado tras el final de la Guerra Mundial. Es cierto que en adelante y como ha sido puesto de manifiesto en distintos trabajos de investigación, se abrieron en el seno de la Asociación criterios políticos diferentes sobre cómo afrontar la realidad española y que el colaboracionismo "evolucionista» de Martín-Artajo o Ruiz-Giménez (y el propio Ángel Herrera), se vió contrarestado con visones más «inmovilistas» defendidas por el propio presidente MartínSánchez. ${ }^{32}$ Pero lo que se ha tratado de demostrar en este artículo, es cómo muchos propagandistas participaron en las estructuras oficiales del nuevo estado desde los primeros momentos del franquismo, sembrando con ello la semilla necesaria que posibilitase su despegue definitivo a partir de 1945. Los propagandistas contribuyeron a la institucionalización del régimen aún sintiéndose vigilados por los elementos proclives a Falange, recelosos de las posiciones legalistas que muchos católicos habían mantenido en la República.

La ACNdeP, fuertemente mermada en el número de sus Centros y miembros por la represión que había sufrido durante la Guerra Civil, se mostró partidaria de cooperar con el estado surgido tras el conflicto. De la mano de Fernando Martín-Sánchez, los propagandistas fueron capaces de reorganizar su estructura organizativa y aunque estatutariamente apolítica - se ha comprobado cómo destacados miembros de la Asociación participaron en la gestación del nuevo modelo político contribuyendo a la vertebración del estado franquista. Los Ministerios de Educación y Justicia, fueron el núcleo de influencia para la labor apostólica de los propagandistas y desde ellos, trataron de influenciar sobre las estructuras nacientes un carácter católico, que había sido mermado durante los años republicanos y que se mantenía en litigio con los elementos próximos al pensamiento falangista. En este sentido, se ha puesto en valor el papel de José Ibáñez Martín quien desde su ministerio, además de la presidencia del CSIC, contribuyó a restructurar las nuevas políticas educativas através de la Ley de Ordenación Universitaria de 1943 y la Ley de Educación Primaria de 1945. Ibáñez-Martín se rodeó de una selección

32 Sobre el papel de los propagandistas en el Estado a comienzos de la década de los 50, puede verse Barreiro Gordillo 2012: 213-242 y Martín Puerta 2005: 22-35. 
de colaboradores en la que tomó especial premimencia Luis Ortiz Muñoz además de un equipo de propagandistas que desde sus puestos como rectores $-y$ también Decanosestaban destinados a tomar la batuta de las Universidades españolas. La cartera de Justicia, se convirtió en otro espacio de actuación para los propagandistas en su tarea institucionalizadora y muy especialmente, a través de los diferentes patronatos organizados para la restructuración política en connivencia con el orden social, bien a través de la infancia, el trabajo o la mujer. Y en este sentido, además de la participación de un elevado porcentaje de propagandistas en las Cortes franquistas como procuradores, se constata especialmente relevante, la importancia del propagandista y catedrático de Derecho, Joaquín Ruiz-Giménez en el proceso de redacción del Fuero de los Españoles de julio de 1945, punto de inflexión en el proceso evolutivo del franquismo. Además, y como se ha tratado de manifestar a lo largo de este trabajo, los hombres de la ACNdeP desempeñaron una tarea principal de cara a dotar de preminencia a los intereses católicos en España y a facilitar una mayor fluidez en las relaciones de las instituciones franquistas con la jerarquía eclesiástica. ${ }^{33}$ En este sentido, su papel en la aprobación de los acuerdos de 1941 se perfiló intenso. Además, aplaudieron y apoyaron el marco de cordialidad, y la voluntad por parte del Estado, de construir un Estado católico.

Por todo ello, y aunque es objetivo afirmar que la influencia de los hombres de la ACNdeP se hace claramente ascendente a partir de la formación del Gobierno de julio de 1945 con la entrada de Martín-Artajo en el Ministerio de Exteriores, podemos aseverar que la participación de los católicos propagandistas en el estado iniciada durante la Guerra Civil, fue el embrión necesario de cara a la configuración de un Estado católico, determinante para su despegue definitivo una vez terminado el conflicto mundial: corporaciones locales, ayuntamientos, organismos políticoculturales y organizaciones estatales, se valieron de los propagandistas para articular una organización estatal proclive a los intereses católicos y a su doctrina social.

\section{BiBLIOGRAFÍA}

Aguilar y Gil Pecharromán, J. 2008. Con permiso de la autoridad. La España de Franco (1939-1975). Madrid: Temas de Hoy.

Alted Vigil, A. 1991. "Bases político ideológicas y jurídicas de la Universidad franquista durante los ministerios de Sáinz Rodríguez y primera época de Ibáñez Martín (1938-1945)», en J. J. Carreras Ares y M. Á. Ruiz Carnicer (eds.), La Universidad española bajo el régimen de Franco (1939-1975): 95-124. Zaragoza: Institución Fernando el Católico.

Andres Gallego, J. y Pazos, A. M. 1999. La Iglesia en la España contemporánea. 1936-1999. Madrid: Encuentro.

Barreiro Gordillo, C. 2010. Historia de la Asociación católica Nacional del Propagandistas. La Presidencia de Fernando Martín-Sánchez (1935-1953). Madrid: CEU-Ediciones.

33 Archivo de la ACNdeP. Carta confidencial de Martín-Sánchez a Ricardo Sánchez de Movellán, del año 1952, informándole acerca de las gestiones realizadas con Ruíz-Giménez para que la Jerarquía designase a algún propagandistas en el Consejo de Educación Nacional, en virtud del derecho de la Iglesia de nombrar a 18 consejeros. Carta de MartínSánchez a Ruiz-Giménez agradeciéndole las gestiones realizadas en el ministerio para incluir a la ACNdeP entre las entidades con capacidad para nombrar representantes en el Consejo de Educación Nacional.
Barreiro Gordillo, C. 2012. "Ángel Herrera y la ACNdeP en la configuración del franquismo», en A. C. Moreno Cantano (ed.), Propagandistas y diplomáticos al servicio de Franco (1936-1945): 213-241. Gijón: Trea Ediciones.

Cárcel, V. 2008. Pío XI entre la República y Franco. Angustia del Papa ante la tragedia española. Madrid: Biblioteca de Autores Cristianos.

Gutiérrez, J. L., Barreiro, C. y Martín Puerta, A. 2010. Historia de la Asociación católica de Propagandistas. Madrid: CEU-Ediciones.

Hermet, G. 1985-1986. Los católicos en la España franquista. Madrid: CIS-Siglo XXI.

Lain Entralgo, P. 2004. Descargo de conciencia (1930-1960). Barcelona: Galaxia Gutemberg.

Larraz, J., López Garrido, P. y Velarde, J. 2011. José Larraz, Política social y bien común. Murcia: Isabor.

Martín Artajo, A. 1950. La política de aislamiento de España seguida por las naciones aliadas durante el quinquenio 1945-1950. Madrid: Oficina de Información Diplomática del Ministerio de Asuntos Exteriores.

Martín de Santa Olalla, P. 2003. De la victoria al Concordato. Las relaciones Iglesia-Estado durante el "Primer Franquismo» (19391953). Barcelona: Laertes.

Martín de Santa Olalla, P. 2012. El Rey, Ia Iglesia y la Transición. Madrid: Sílex.

Martín Puerta, A. 2005. "Los propagandistas en los primeros años 50». Aportes: Revista de Historia Contemporánea 57: 22-35.

Martín Puerta, A. 2015. «La ACNdeP durante la fase central del régimen de Franco». Hispania Sacra 67 (135): 303-338.

Mayordomo Pérez, A. (coord.). 1999. Estudios sobre la política educativa durante el franquismo. Valencia: Universidad de Valencia.

Montero, M. 1993. Historia de la A.C. N. de P. La construcción del Estado Confesional. 1936-1945. Navarra: Eunsa.

Montero, M. 2001. Cultura y comunicación al servicio de un régimen. Historia de la ACNdeP entre 1945 y 1959. Pamplona: Eunsa.

Muñoz Soro, J. 2006. «Joaquín Ruiz-Giménez o el católico total (apuntes para una biografía política e intelectual». Pasado y Memoria. Revista de Historia Contemporánea 5: 259-288.

Orella Martínez, J. L. 2001. La formación del Estado Nacional. Madrid: Actas.

Payne, S. 1984. El catolicismo español. Barcelona: Planeta.

Payne, S. 1987. El régimen de Franco. 1936-1975. Madrid.

Payne, S. 1998. Franco y José Antonio. Barcelona: Planeta.

Pérez Crespo, A. 2013. Historia del Centro de Murcia de la Asociación Católica de Propagandistas (ACdP). De 1926-2011.: Madrid: CEUEdiciones.

Peset, M. 1991. "La Ley de Ordenación Universitaria de 1943», en J. J. Carreras Ares y M. Á. Ruiz Carnicer (eds.), La Universidad española bajo el régimen de Franco (1939-1975): 125-158. Zaragoza: Institución Fernando el Católico.

Pizarroso Quintero, A. 2009. Diplomáticos, propagandistas y espías. Estados Unidos y España en la Segunda Guerra Mundial. Información y Propaganda. Madrid: CSIC.

Portero, F. 1989. Franco aislado. La cuestión española (1945-1950). Madrid: Aguilar.

Redondo, G. 1999. Política, cultura y sociedad en la España de Franco. 1939-1975. Tomo I. La configuración del Estado Español, nacional y católico (1939-1947). Pamplona: Eunsa.

Robles Muñoz, C. 2009. "En favor de las víctimas de la guerra». Hispania Sacra 61 (124): 691-753.

Ruiz Rico, J. J. 1977. El papel político de la Iglesia católica en la España de Franco (1936-1971). Madrid: Tecnos.

Sánchez Garrido, P. 2017. «Génesis e identidad del grupo fundacional de la ACN de JP (1904-1909)». Hispania Sacra 69 (139): 389-400.

Sánchez Recio, G. (coord.). 2005. La Internacional Católica. Pax Romana en la política europea de posguerra. Madrid: Biblioteca Nueva.

Tusell, J. 1984. Franco y los católicos. La política interior española entre 1945 y 1957 . Madrid: Alianza. 